\title{
Inhomogeneous diophantine approximation on polynomials in $\mathbb{Q}_{p}$
}

\author{
by
}

\section{Bernik (Minsk), H. DiCkinson (York) and J. YuAn (Xi'an)}

In 1932 Mahler [6], following his fundamental study of the theory of transcendental numbers, formulated the conjecture that for any $\varepsilon>0$ the inequality

$$
|P(x)|<H(P)^{-n-\varepsilon}
$$

has at most a finite number of solutions in integer polynomials $P$ of degree $n$ for almost all $x \in \mathbb{R}$, where $H(P)$ is the height of $P$ (that is, $H(P)$ is the maximum of the moduli of the coefficients of $P$ ). This was proved by Sprindžuk [7]. In 1965 Sprindžuk [8] also considered the $p$-adic analogue of Mahler's conjecture and proved Theorem 1 below. Throughout this paper $\mathbb{Q}_{p}$ denotes the $p$-adic field with $p$-adic metric $|\cdot|_{p}$ and $\mathbb{Z}_{p}=\left\{w \in \mathbb{Q}_{p}:|w|_{p} \leq 1\right\}$ denotes the $p$-adic integers. A ball $B(a ; \delta)$ in $\mathbb{Q}_{p}$ is defined as

$$
B(a ; \delta)=\left\{x \in \mathbb{Q}_{p}:|x-a|_{p} \leq \delta\right\} .
$$

It has diameter $\operatorname{diam}(B)=\delta$ and measure $\nu(B)=\delta$, where $\nu$ is the unique Haar measure on the locally compact abelian group $\mathbb{Q}_{p}$ such that $\nu\left(\mathbb{Z}_{p}\right)=1$.

Theorem 1 (Mahler-Sprindžuk). The inequality

$$
|P(w)|_{p}<H(P)^{-n-1-\varepsilon}
$$

has only a finite number of solutions in rational integer polynomials $P$ of degree $n$ for almost all $w \in \mathbb{Q}_{p}$ (with respect to Haar measure).

A proof of this theorem can be found in [8, Part II, Chapter 2]. Similar inhomogeneous problems can be considered and for $n=1$ the following inhomogeneous question has been solved [5, Chapter 2, Theorem 4.11]. Indeed it is not difficult to prove that for every $d \in \mathbb{Q}_{p}$ and almost all $w \in \mathbb{Q}_{p}$ the inequality

$$
\left|q_{1} w+q_{0}+d\right|_{p}<|q|^{-2-\varepsilon}
$$

1991 Mathematics Subject Classification: Primary 11J61; Secondary 11J54. 
where $q=\max \left(q_{0}, q_{1}\right)$, has only a finite number of solutions in rational integers $q_{1}$ and $q_{0}$ for every $\varepsilon>0$.

Inhomogeneous questions are rather different in character to the homogeneous ones in that they concern questions of how points are distributed rather than how close it is possible to get to the integers. It is often the case that, due to the properties of the $p$-adic metric, a problem looked at in the $p$-adic setting is easier to solve than a similar one considered for $\mathrm{Eu}$ clidean space. In this article we will prove the inhomogeneous analogue of Theorem 1, that is, we will consider inequality (1) for polynomials of higher degree.

TheOREM 2. For every $d \in \mathbb{Q}_{p}$ the inequality

$$
|P(w)+d|_{p}<H(P)^{-n-1-\varepsilon}
$$

has only a finite number of solutions in rational integer polynomials $P$ of degree $n$ for almost all $w \in \mathbb{Q}_{p}$.

It is hoped that the methods used in this paper can be generalised to prove a similar result for the usual Euclidean metric.

In what follows the Vinogradov symbols $\ll$ and $\gg$ will be used to avoid specifying unimportant constants $(A \ll B$ means that there exists a constant $c$ such that $A \leq c B$ with a similar definition for $A \gg B$ ); if $A \ll B$ and $A \gg B$ then we write $A \asymp B$.

The proof of Theorem 2 will involve repeated applications of the BorelCantelli lemma; that is, if the sum of measures of a sequence of sets converges then the measure of the set of points lying in infinitely many such sets is zero. Before proving Theorem 2 some preliminary results are obtained.

LEMMA 1. Let

$$
P(w)=a_{n} w^{n}+\ldots+a_{1} w+a_{0}
$$

be a polynomial with rational integer coefficients. Then

$$
\max _{0 \leq m \leq n}|P(m)| \gg \max _{0 \leq i \leq n}\left|a_{i}\right| .
$$

We denote the smallest $m$ for which (4) is true by $m_{0}$. This is Lemma 7 of $[7$, p. 19].

Lemma 2. Let $P$ be a polynomial of the form (3) with $\left|a_{n}\right|_{p}>c_{1}$, where $c_{1}$ is a constant depending only on $n$. Then

$$
\left|\kappa_{i}\right|_{p} \leq \max \left(1 / c_{1}, 1\right)
$$

for every root $\kappa_{i}, i=1, \ldots, n$, of $P$. 
Proof. If $\left|\kappa_{i}\right|_{p} \leq 1$ then inequality (5) is true. Assume therefore that $\left|\kappa_{i}\right|_{p}>1$. Evidently $a_{n} \kappa_{i}^{n}=-a_{n-1} \kappa_{i}^{n-1}-\ldots-a_{1} \kappa_{i}-a_{0}$, so that

$$
\kappa_{i}=-\frac{a_{n-1}}{a_{n}}-\ldots-\frac{a_{1}}{a_{n} \kappa_{i}^{n-2}}-\frac{a_{0}}{a_{n} \kappa_{i}^{n-1}}
$$

further implying that

$$
\left|\kappa_{i}\right|_{p} \leq \max _{0 \leq j \leq n-1}\left|\frac{a_{j}}{a_{n} \kappa_{i}^{n-1-j}}\right|_{p} \leq \frac{1}{c_{1}}
$$

If inequality (2) holds infinitely often for a set of positive measure it can be readily verified that the set of solutions of the inequality

$$
|Q(w)|_{p}<H(P)^{-n-1-\varepsilon}
$$

where $Q(w)=w^{n}\left(P\left(w^{-1}+m_{0}\right)+d\right)$ also has positive measure, where $m_{0}$ is the fixed integer from Lemma 1 (see [1, Lemma 5$]$ for details). It is easy to show that $Q$ has the form

$$
Q(w)=\left(P\left(m_{0}\right)+d\right) w^{n}+b_{n-1} w^{n-1}+\ldots+b_{1} w+b_{0}
$$

where $b_{i} \in \mathbb{Z}$ for $i=0, \ldots, n-1$. If $\left|P\left(m_{0}\right)+d\right|_{p}$ is very small then consider instead the case $P\left(m_{0}\right)+d+1$ (or $P\left(m_{0}\right)+d-1$ ) which will have $p$-adic modulus 1 . If the set of $w$ for which inequality (2) holds for $d+1$ for infinitely many $P$ is of measure zero then so is the set of $w$ for which inequality (2) holds for $d$ as otherwise we obtain a contradiction (replacing $P(w)+d$ by $(P(w)-1)+d+1)$. Hence, we may assume without loss of generality that $\left|b_{n}\right|_{p}=\left|P\left(m_{0}\right)+d\right|_{p}>c_{2}$, where $c_{2}$ is a constant depending only on $n$ and $d$; therefore the roots of $Q$ are bounded from Lemma 2. Thus, instead of inequality (2), we can consider the inequality

$$
\left|P_{d}(w)\right|_{p}<H\left(P_{d}\right)^{-n-1-\varepsilon},
$$

where

$$
P_{d}(w)=\left(a_{n}+d\right) w^{n}+\ldots+a_{1} w+a_{0}=N_{1} w^{n}+a_{n-1} w^{n-1}+\ldots+a_{0}
$$

and the roots of $P_{d}$ lie in the ball $|w|_{p} \ll 1$. Note that $N_{1}$ is not necessarily an integer. Let

$$
\mathcal{P}_{n}(N)=\left\{P_{d}(w): a_{n}=N,\left|a_{i}\right| \ll N,\left|\kappa_{i}\right|_{p} \ll 1\right\}
$$

where $N$ depends on the height of the polynomial $P$ associated with $P_{d}$.

Let $\varepsilon>0$ be sufficiently small, $T$ be a large fixed number depending on $\varepsilon$ and let $\varepsilon_{1}=1 / T\left(\varepsilon_{1}\right.$ is much smaller than $\varepsilon$ ). The roots $\kappa_{1}, \ldots, \kappa_{n}$ of the polynomials $P_{d}$ can be ordered (by changing indices if necessary) so that

$$
\left|\kappa_{1}-\kappa_{2}\right|_{p} \leq\left|\kappa_{1}-\kappa_{3}\right|_{p} \leq \ldots \leq\left|\kappa_{1}-\kappa_{n}\right|_{p} .
$$

For each $i=2, \ldots, n$ define $\mu_{i}=\mu_{i}\left(P_{d}\right)$ in $\mathbb{R}$ by the relation

$$
\left|\kappa_{1}-\kappa_{i}\right|_{p}=H\left(P_{d}\right)^{-\mu_{i}}
$$


and integers $l_{i}=l_{i}\left(P_{d}\right)$ as follows: if $\mu_{i}>n$ write $l_{i}=\infty$; otherwise $l_{i}$ is the unique integer such that

$$
\left(l_{i}-1\right) / T \leq \mu_{i}<l_{i} / T .
$$

It is easy to show that $l_{i} \geq-1$ for large $N$ since $\left|\kappa_{1}-\kappa_{i}\right|_{p} \ll 1$ and that $l_{2} \geq$ $l_{3} \geq \ldots \geq l_{n}$. Thus every number $l_{i}$ can take the values $-1,0,1, \ldots, n T, \infty$. Since the number of different vectors $\mathbf{l}=\left(l_{2}, \ldots, l_{n}\right)$ is at most $(n T+3)^{n-1}$, we can fix the vector $\mathbf{l}$ and the class $\mathcal{P}_{n}(\mathbf{l})$ as the set of polynomials with $\mathbf{l}\left(P_{d}\right)=\mathbf{l}$. We define $\mathcal{P}_{n}(N, \mathbf{l})$ as the set $\mathcal{P}_{n}(N) \cap \mathcal{P}_{n}(\mathbf{l})$ so that

$$
\mathcal{P}_{n}(\mathbf{l})=\bigcup_{N=1}^{\infty} \mathcal{P}_{n}(N, \mathbf{l})
$$

It is also useful to define the numbers $r_{i}$ as

$$
r_{i}=\frac{l_{i+1}+\ldots+l_{n}}{T}, \quad i=1, \ldots, n-1 .
$$

Finally we define the set $S\left(\kappa_{i}\right)$ as follows:

$$
S\left(\kappa_{i}\right)=\left\{w \in \mathbb{Q}_{p}: \min _{1 \leq j \leq n}\left|w-\kappa_{j}\right|_{p}=\left|w-\kappa_{i}\right|_{p}\right\} .
$$

Thus $S\left(\kappa_{i}\right)$ is the set of $w$ which are closer to $\kappa_{i}$ than any other root. Plainly $\bigcup S\left(\kappa_{i}\right)=\mathbb{Q}_{p}$. It is possible that some of the $S\left(\kappa_{i}\right)$ are empty.

Now we need some subsidiary results regarding polynomials and their derivatives. For simplicity the lemmas are set up using $\kappa_{1}$, they could also have been done for any other root $\kappa_{i}$.

LEMMA 3. Let $P$ be a polynomial with distinct roots and $w$ be in $S\left(\kappa_{1}\right)$. Then

and

$$
\left|w-\kappa_{1}\right|_{p} \leq \frac{|P(w)|_{p}}{\left|P^{\prime}\left(\kappa_{1}\right)\right|_{p}}
$$

for $j=2, \ldots, n$.

Thus as $\left|\kappa_{i}\right|_{p} \ll 1$ inequality (6) can only hold on a ball $A$ such that $\operatorname{diam}(A) \ll 1$. Hence from now on we assume without loss of generality that $w \in A$.

Proof (of Lemma 3 ). If $w \in S\left(\kappa_{1}\right)$, then

$$
\begin{aligned}
\left|\kappa_{1}-\kappa_{i}\right|_{p} & =\left|\left(w-\kappa_{i}\right)-\left(w-\kappa_{1}\right)\right|_{p} \\
& \leq \max \left(\left|w-\kappa_{i}\right|_{p},\left|w-\kappa_{1}\right|_{p}\right)=\left|w-\kappa_{i}\right|_{p} .
\end{aligned}
$$


From the identity

$$
\left|w-\kappa_{1}\right|_{p}=\frac{|P(w)|_{p}}{\left|a_{n}\left(w-\kappa_{2}\right) \ldots\left(w-\kappa_{n}\right)\right|_{p}},
$$

and (11) we have

$$
\left|w-\kappa_{1}\right|_{p} \leq \frac{|P(w)|_{p}}{\left|a_{n}\left(\kappa_{1}-\kappa_{2}\right) \ldots\left(\kappa_{1}-\kappa_{n}\right)\right|_{p}}=\frac{|P(w)|_{p}}{\left|P^{\prime}\left(\kappa_{1}\right)\right|_{p}} .
$$

Also

$$
\begin{aligned}
\left|w-\kappa_{1}\right|_{p}^{j} & \leq\left|\left(w-\kappa_{1}\right) \ldots\left(w-\kappa_{j}\right)\right|_{p}=\frac{|P(w)|_{p}}{\left|a_{n}\left(w-\kappa_{j+1}\right) \ldots\left(w-\kappa_{n}\right)\right|_{p}} \\
& \leq \frac{|P(w)|_{p}}{\left|a_{n}\left(\kappa_{1}-\kappa_{j+1}\right) \ldots\left(\kappa_{1}-\kappa_{n}\right)\right|_{p}} \\
& =\frac{|P(w)|_{p}}{\left|P^{\prime}\left(\kappa_{1}\right)\right|_{p}}\left|\left(\kappa_{1}-\kappa_{2}\right) \ldots\left(\kappa_{1}-\kappa_{j}\right)\right|_{p}
\end{aligned}
$$

from which we obtain (10).

Lemma 4. Let $P_{d} \in \mathcal{P}_{n}(N, \mathrm{l})$. Then if $l_{i} \neq \infty$ for any $i>j$

$$
\left|P_{d}^{(j)}\left(\kappa_{1}\right)\right|_{p} \ll\left|\left(\kappa_{1}-\kappa_{j+1}\right) \ldots\left(\kappa_{1}-\kappa_{n}\right)\right|_{p} \ll N^{-r_{j}+(n-j) \varepsilon_{1}}
$$

for $j=1, \ldots, n-1$. If on the other hand $l_{2}=l_{3}=\ldots=l_{i}=\infty$ for some $i>j$ then

$$
\left|P_{d}^{(j)}\left(\kappa_{1}\right)\right|_{p} \ll N^{-(i-j) n-r_{i}+(n-i) \varepsilon_{1}}
$$

(define $r_{n}$ to be 0 ).

Proof. As $P_{d}(w)=N_{1}\left(w-\kappa_{1}\right) \ldots\left(w-\kappa_{n}\right), P_{d}^{(j)}(w)$ is the sum of terms $c_{3} N_{1}\left(w-\kappa_{i_{1}}\right) \ldots\left(w-\kappa_{i_{n-j}}\right)$, where $\left\{i_{1}, \ldots, i_{n-j}\right\}$ is any subset of $\{1, \ldots, n\}$ and $c_{3}$ is a constant depending on $j$. If $\kappa_{1}$ is substituted for $w$ then most of the terms vanish and $P_{d}^{(j)}\left(\kappa_{1}\right)$ is the sum of terms $c_{3} N_{1}\left(\kappa_{1}-\kappa_{i_{1}}\right) \ldots\left(\kappa_{1}-\right.$ $\left.\kappa_{i_{n-j}}\right)$. Owing to the root ordering the term with largest $p$-adic norm is $c_{3} N_{1}\left(\kappa_{1}-\kappa_{j+1}\right) \ldots\left(\kappa_{1}-\kappa_{n}\right)$. Therefore

$\left|P_{d}^{(j)}\left(\kappa_{1}\right)\right|_{p}=\left|c_{3} N_{1} \sum_{\left\{i_{1}, \ldots, i_{n-j}\right\} \subset\{1, \ldots, n\}}\left(\kappa_{1}-\kappa_{i_{1}}\right)\left(\kappa_{1}-\kappa_{i_{2}}\right) \ldots\left(\kappa_{1}-\kappa_{i_{n-j}}\right)\right|_{p}$,

which, as $\left|N_{1}\right|_{p} \ll 1$, is

$$
\ll\left|\left(\kappa_{1}-\kappa_{j+1}\right) \ldots\left(\kappa_{1}-\kappa_{n}\right)\right|_{p}=N^{-\sum_{i=j+1}^{n} \mu_{i}}
$$

from equation (8). Using (9) this gives (12); if $l_{i}=\infty$ for some $i$ then $\mu_{t} \geq n$ for $t=2, \ldots, i$, which gives (13).

The next lemma is proved in [3] which is in Russian and difficult to obtain. For convenience we refer the reader to Lemma 12 of [2], in which 
the same lemma is proved for the real case. A sketch of the main ideas will follow the statement.

LEMMA 5. Let $\delta>0, \eta>0$ be real numbers, $n \geq 2$ a natural number and $N=N(\delta, n)$ a sufficiently large real number. Further let $P, Q$ in $\mathbb{Z}[w]$ be two relatively prime polynomials of degree at most $n$ with $\max (H(P), H(Q)) \leq$ $N$. Let $B\left(\xi ; p^{-s}\right)$ be a ball in $\mathbb{Q}_{p}$ where $s$ is defined by the inequalities $p^{-s} \leq$ $N^{-\eta}<p^{-s+1}$. If there exists $\tau>0$ such that for all $w \in B\left(\xi ; p^{-s}\right)$,

$$
\max \left(|P(w)|_{p},|Q(w)|_{p}\right)<N^{-\tau},
$$

then

$$
\tau+2 \max (\tau-\eta, 0)<2 n+\delta .
$$

With reference to the notation in Lemma 12 of [2], for our case $\mu=0$. By following the proof exactly it can be shown that for $w \in \nu_{1}(P)$,

$$
\left|w-\kappa_{1}(P)\right|_{p} \ll N^{-\left(\tau-r_{j}(P)\right) / j},
$$

and for $w \in \nu_{1}(Q)$,

$$
\left|w-\kappa_{1}(Q)\right|_{p} \ll N^{-\left(\tau-r_{j}(Q)\right) / j},
$$

for $j=1, \ldots, n$. Let the minimum of the right hand sides of these two equations be at $j=j^{\prime}$ and $j=j^{\prime \prime}$ respectively. Then following the arguments of [2] to obtain results analogous to inequalities (28) to (36) of [2] we have for $i<j^{\prime}$ and $k<j^{\prime \prime}$ respectively

$$
l_{i+1}(P) \geq \frac{\tau-r_{j^{\prime}}(P)}{j^{\prime}} \text { and } \quad l_{k+1}(Q) \geq \frac{\tau-r_{j^{\prime \prime}}(Q)}{j^{\prime \prime}} .
$$

Also for $i>j^{\prime}$ and $k>j^{\prime \prime}$ respectively

$$
l_{i}(P) \leq \frac{\tau-r_{i}(P)}{i} \leq \frac{\tau-r_{j^{\prime}}(P)}{j^{\prime}}, \quad l_{k}(Q) \leq \frac{\tau-r_{i}(Q)}{i} \leq \frac{\tau-r_{j^{\prime \prime}}(Q)}{j^{\prime \prime}},
$$

and finally we obtain

$$
\eta \geq \max _{1 \leq i, j \leq n}\left\{\frac{\tau-r_{i}(P)}{i}, \frac{\tau-r_{j}(Q)}{j}\right\} .
$$

Using these estimates it is not difficult to obtain the following inequality analogous to (42) of [2]:

$$
\prod_{1 \leq i \leq j^{\prime \prime}} \prod_{1 \leq j \leq j^{\prime}}\left|\kappa_{i}(Q)-\kappa_{j}(P)\right|_{p} \ll N^{-j^{\prime \prime}\left(\tau-r_{j^{\prime}}(P)\right)}
$$

for $j^{\prime}, j^{\prime \prime} \geq 1$. Using the same arguments that follow inequality (42) until inequalities (45) in [2], it can be shown that $1 \ll N^{2 n-j^{\prime \prime} \tau}$, i.e., that $j^{\prime \prime} \tau \leq$ $2 n+\delta$. If $j^{\prime \prime} \geq 3$ then the lemma is proved. Thus we only need to obtain better estimates for the cases $j^{\prime \prime}=1$ and $j^{\prime \prime}=2$ as in [2]. The line of reasoning is the same as in that paper and will therefore not be given. 
Lemma 6. If $P_{1}, \ldots, P_{k}$ are given polynomials then

$$
H\left(P_{1} \ldots P_{k}\right) \asymp H\left(P_{1}\right) \ldots H\left(P_{k}\right) .
$$

This is Lemma 8 from [8, Chapter 1]. A refined version with explicit constants was obtained by Gel'fond in [4].

Finally, the following lemma is required regarding reducible polynomials.

Lemma 7. Let $P$ be a reducible polynomial with rational integer coefficients and degree at most $n$ and let $\delta$ be any positive number. Then for almost all $w \in \mathbb{Q}_{p}$ the inequality

$$
|P(w)|_{p}<H(P)^{-n-\delta}
$$

has only a finite number of solutions.

Pr o of. The argument is similar to one used by Sprindžuk in [8] which uses the sup norm rather than the $p$-adic metric. Any reducible rational integer polynomial $P$ of degree at most $n$ can be expressed as a product

$$
P(w)=P_{1}(w) P_{2}(w)
$$

where $P_{1}$ and $P_{2}$ are rational integer polynomials of degree at most $n-1$. Suppose that (15) holds on a set $S$ say of positive measure for infinitely many reducible polynomials $P$ of degree at most $n$. Then for any $\delta>0$ and each $w \in S$, there exist infinitely many $P_{1}, P_{2}$ such that, by Lemma 6 ,

$$
\left|P_{1}(w) P_{2}(w)\right|_{p}<H(P)^{-n-\delta} \leq c H\left(P_{1}\right)^{-n-\delta} H\left(P_{2}\right)^{-n-\delta}
$$

for some constant $c>0$. Without loss of generality we can suppose that $w$ is transcendental so that $P(w) \neq 0$ for any $P$. By Theorem 1, for Haar almost all $w \in S$, the inequalities

$$
\left|P_{1}(w)\right|_{p} \geq H\left(P_{1}\right)^{-n-\delta^{\prime}}, \quad\left|P_{2}(w)\right|_{p} \geq H\left(P_{2}\right)^{-n-\delta^{\prime}}
$$

hold for all $\delta^{\prime}>0$ and all but finitely many $P_{1}, P_{2}$. Choose one such $w$. For those polynomials $P_{1}, \ldots, P_{l}$ for which the above equation does not hold let $c(w)=\min \left\{P_{1}(w), \ldots, P_{n}(w)\right\}$. Then

$$
\left|P_{1}(w)\right|_{p} \geq c(w) H\left(P_{1}\right)^{-n-\delta^{\prime}}, \quad\left|P_{2}(w)\right|_{p} \geq c(w) H\left(P_{2}\right)^{-n-\delta^{\prime}}
$$

for all $P_{1}, P_{2}$. On multiplying, we get

$$
\left|P_{1}(w) P_{2}(w)\right|_{p} \geq H\left(P_{1}\right)^{-n-\delta^{\prime}} H\left(P_{2}\right)^{-n-\delta^{\prime}}
$$

for all pairs $P_{1}, P_{2}$, which contradicts $w$ being in $S$ for $\delta^{\prime}<\delta$.

Proof of Theorem 2. The proof of the theorem is in two parts. A class $\mathcal{P}_{n}(\mathbf{l})$ will be described as being of the first or second kind according as

$$
l_{2} T^{-1}+r_{1} \leq n \text { or }>n .
$$

For classes of the first kind $\left(l_{2} T^{-1}+r_{1} \leq n\right)$ we consider the class of polynomials $\mathcal{P}_{n}(N, \mathbf{l})$ and prove the theorem with the help of Sprindžuk's method 
of essential and inessential domains [8, Part II, Chapter 2]. The proof for the inhomogeneous case is the same as that for the homogeneous case in [8] and will not be repeated. This case is where the roots of the polynomials are "far" apart so that the derivative at the roots is not too small.

For classes of the second kind

$$
l_{2} T^{-1}+r_{1}>n .
$$

In this case the roots can be very close together. This is the major difference between the inhomogeneous and homogeneous questions. When considering the homogeneous case one can use the discriminant to show that the roots are always a certain distance apart (with respect to the height) and that there are only finitely many bounded vectors 1 . Because the polynomials $P_{d}$ are no longer integer polynomials due to the first coefficient being $N+d$ it is not possible to use the discriminant, and the roots can be arbitrarily close together.

First the ball $A$ is divided into $\ll N^{\sigma}$ subballs $S_{k}$ having the same diameter $N^{-\sigma}$ where

$$
\sigma=\max _{1 \leq i \leq n-1} \frac{n-r_{i}}{i}+n \varepsilon_{1},
$$

for $l_{i} \neq \infty$ for all $i=2, \ldots, n$. In the event that $l_{i}=\infty$ for all $i$, we have $\sigma=1+\varepsilon /(2 n)$; this case will be dealt with at the end. Assume that the maximum on the right hand side of (17) is at $i=j_{0}$ and define

$$
\mathcal{P}_{n}^{t}(\mathbf{l})=\bigcup_{2^{t} \leq N<2^{t+1}} \mathcal{P}_{n}(N, \mathbf{l}) .
$$

Note that $j_{0} \geq 2$ since if $j_{0}=1$, then (16) and (17) give a contradiction. The polynomial $P_{d}$ will be said to belong to the ball $S_{k}$ if there exists $w \in S_{k}$ such that (6) holds. Assume that there are no more than $2^{t\left(1 / j_{0}-\varepsilon / 2\right)}$ polynomials $P_{d} \in \mathcal{P}_{n}^{t}(\mathbf{l})$ belonging to each ball $S_{k}$. Then the number $K(\mathcal{P})$ of polynomials in the class $\mathcal{P}_{n}^{t}(\mathbf{l})$ satisfying $(6)$ is

$$
K(\mathcal{P}) \ll 2^{t\left(\left(n-r_{j_{0}}\right) / j_{0}+n \varepsilon_{1}\right)} 2^{t\left(1 / j_{0}-\varepsilon / 2\right)} .
$$

By Lemma 3 the measure of the set $I\left(P_{d}\right)$ for which inequality (6) holds for a fixed polynomial $P_{d}$ is

$$
\nu\left(I\left(P_{d}\right)\right) \ll 2^{-t\left(\left(n+1-r_{j_{0}}+\varepsilon\right) / j_{0}\right)},
$$

whence

$$
K(\mathcal{P}) \nu\left(I\left(P_{d}\right)\right) \ll 2^{-t\left(\varepsilon / 2+\varepsilon / j_{0}-n \varepsilon_{1}\right)} \ll 2^{-t \varepsilon / 2} .
$$

Since the series $\sum_{t} 2^{-t \varepsilon / 2}$ converges the proof can be completed by using the Borel-Cantelli lemma.

Now, we assume that there are more than $2^{t\left(1 / j_{0}-\varepsilon / 2\right)}$ polynomials in $\mathcal{P}_{n}^{t}(\mathbf{l})$ belonging to some ball $S$. The Taylor series for the polynomials $P_{d}$ 
belonging to $S$ in the neighbourhood of the root $\kappa_{1}=\kappa_{1}\left(P_{d}\right)$ is

$$
\begin{aligned}
P_{d}(w)= & P_{d}^{\prime}\left(\kappa_{1}\right)\left(w-\kappa_{1}\right)+\ldots \\
& \ldots+\frac{P_{d}^{(n-1)}\left(\kappa_{1}\right)}{(n-1) !}\left(w-\kappa_{1}\right)^{n-1}+\frac{P_{d}^{(n)}\left(\kappa_{1}\right)}{n !}\left(w-\kappa_{1}\right)^{n} .
\end{aligned}
$$

As there exists a point $w_{0} \in S$ such that $\left|P_{d}\left(w_{0}\right)\right|_{p}<2^{-t(n+1+\varepsilon)}$ we deduce from (10) that

$$
\left|w_{0}-\kappa_{1}\right|_{p} \ll 2^{-t\left(\left(n+1-r_{j_{0}}+\varepsilon\right) / j_{0}\right)} .
$$

For each $w \in S$ we have $\left|w-w_{0}\right|_{p}<\operatorname{diam}(S)$ and by (21),

$$
\left|w-\kappa_{1}\right|_{p} \leq \max \left(\left|w-w_{0}\right|_{p},\left|w_{0}-\kappa_{1}\right|_{p}\right) \ll 2^{-t\left(\left(n-r_{j_{0}}\right) / j_{0}+n \varepsilon_{1}\right)} .
$$

From (20),

$$
\left|P_{d}(w)\right|_{p} \ll \max _{1 \leq j \leq n}\left|P_{d}^{(j)}\left(\kappa_{1}\right)\left(w-\kappa_{1}\right)^{j}\right|_{p},
$$

and together, Lemma 4 and (22) imply that if $l_{i} \neq \infty$ for some $i$, then

$$
\begin{aligned}
\left|P_{d}^{(j)}\left(\kappa_{1}\right)\left(w-\kappa_{1}\right)^{j}\right|_{p} & \ll 2^{t\left(-r_{j}+(n-j) \varepsilon_{1}\right)} 2^{-t j\left(\left(n-r_{j_{0}}\right) / j_{0}+n \varepsilon_{1}\right)} \\
& \leq 2^{t\left(-r_{j}+(n-j) \varepsilon_{1}\right)} 2^{-t j\left(\left(n-r_{j}\right) / j+n \varepsilon_{1}\right)} \\
& \ll 2^{-t\left(n+\varepsilon_{1}\right)} .
\end{aligned}
$$

The last term in the Taylor expansion can be estimated using (10). For the case of $l_{2}, \ldots, l_{i}$ being infinity, (13) can be used to obtain the same (in fact better) estimate. Thus, for $w \in S$,

$$
\left|P_{d}(w)\right|_{p} \ll 2^{-t\left(n+\varepsilon_{1}\right)} .
$$

Let the polynomials belonging to $S$ be denoted by $P_{1}, \ldots, P_{K}$ where $K>2^{t\left(1 / j_{0}-\varepsilon / 2\right)}$, and define the distinct rational integer polynomials $R_{i}(w)$ for $i=1, \ldots, K-1$ by

$$
R_{1}(w)=P_{2}(w)-P_{1}(w), \quad \ldots, \quad R_{K-1}(w)=P_{K}(w)-P_{1}(w) .
$$

These have the form

$$
R_{i}(w)=a_{n_{i}} w^{n}+\ldots+a_{1_{i}} w+a_{0_{i}}
$$

with $\left|a_{k_{i}}\right|<2^{t+2}$ for $i=1, \ldots, K-1$ and $k=0,1, \ldots, n$ and obey the inequality

$$
\left|R_{i}(w)\right|_{p} \ll 2^{-t\left(n+\varepsilon_{1}\right)} .
$$

There are three different subcases to consider.

CASe 1. Suppose that for each $i, R_{i}(w)=b_{i} R(w)$ with $b_{i} \in \mathbb{Z}$. Since the $R_{i}$ are all different so are the $b_{i}$. Let $b$ be the maximum of the $b_{i}$, so that $|b| \geq$ $K / 2$. Then the polynomial $Q(w)=b R(w)$ has height $H(Q)=|b| H(R)$. Thus $H(R)=H(Q) /|b|<H(Q) 2^{-t\left(1 / j_{0}-\varepsilon / 2\right)+1}$ and since $H(Q) \leq 2 H\left(P_{i}\right) \leq 2^{t+2}$ 
for some $1 \leq i \leq K$ this implies that $H(R) \ll 2^{t\left(1-1 / j_{0}+\varepsilon / 2\right)}$. Therefore $2^{-t} \ll H(R)^{-1 /\left(1-1 / j_{0}+\varepsilon / 2\right)}$ and inequality (24) implies that

$$
|R(w)|_{p} \ll H(R)^{-\mu},
$$

where

$$
\mu=\frac{n+\varepsilon_{1}}{1-1 / j_{0}+\varepsilon / 2} \geq \frac{n+\varepsilon_{1}}{1-1 / n+\varepsilon / 2}>n+1 .
$$

By Theorem 1 the set of $w$ for which there are infinitely many polynomials $R$ satisfying (25) has measure zero.

CASE 2. Suppose now that some of the polynomials $R_{i}$ are reducible. Then, Lemma 7 and (24) show that the set of $w$ for which there exist infinitely many such polynomials is of measure zero.

CASE 3. Suppose that all the polynomials $R_{i}$ are irreducible and that at least two are relatively prime (otherwise use Case 1 ). Then Lemma 5 can be used on two of the polynomials, $R_{1}$ and $R_{2}$ say. Here

$$
\tau=n+\varepsilon_{1}, \quad \eta=\sigma=\frac{n-r_{j_{0}}}{j_{0}}+n \varepsilon_{1},
$$

and by Lemma 5 ,

$$
n+\varepsilon_{1}+2 \max \left(n+\varepsilon_{1}-\frac{n-r_{j_{0}}}{j_{0}}-n \varepsilon_{1}, 0\right)<2 n+\delta .
$$

If $j_{0} \geq 3$ then the inequality (26) cannot hold for sufficiently small $\delta$ since $r_{j_{0}} \geq 0$. If $j_{0}=2$ a more accurate estimate of (23) (the estimate for the term $P_{d}^{\prime}\left(\kappa_{1}\right)\left|w-\kappa_{1}\right|$ comes from Lemma 3$)$ gives that

$$
\left|P_{d}(w)\right|_{p} \ll 2^{-t\left(n+n \varepsilon_{1}\right)}
$$

further implying that

$$
\left|R_{i}(w)\right|_{p} \ll 2^{-t\left(n+n \varepsilon_{1}\right)} .
$$

Now $\tau=n+n \varepsilon_{1}$ and inequality (26) will not hold if $\delta<n \varepsilon_{1}$.

Finally, the case $l_{2}=\ldots=l_{n}=\infty$ is considered. Let $\sigma=1+\varepsilon /(2 n)$. The argument of the proof is exactly the same so only the differences will be pointed out. First assume that there are no more than $2^{t(1 / n-\varepsilon / n)}$ polynomials $P_{d} \in \mathcal{P}_{n}^{t}(\mathbf{l})$. Then quantity (19) becomes

$$
K(\mathcal{P}) \ll 2^{t(1+\varepsilon /(2 n))} 2^{t(1 / n-\varepsilon / n)} .
$$

The measure of the set $I\left(P_{d}\right)$ for which inequality (6) holds for a fixed polynomial $P_{d}$ is

$$
\nu\left(I\left(P_{d}\right)\right) \ll 2^{-t(1+(1+\varepsilon) / n)}
$$

giving that 


$$
K(\mathcal{P}) \nu\left(I\left(P_{d}\right)\right) \ll 2^{-t(2 \varepsilon / n-\varepsilon /(2 n))}=2^{-3 t \varepsilon /(2 n)},
$$

which converges when summed over $t$.

Now assume that there are more than $K=2^{t(1 / n-\varepsilon / n)}$ polynomials in $\mathcal{P}_{n}^{t}(\mathbf{l})$ belonging to some ball $S$, and making the necessary modifications, follow the proof until inequality (21) which becomes

$$
\left|w_{0}-\kappa_{1}\right|_{p} \ll 2^{-t(1+(1+\varepsilon) / n)}
$$

and inequalities (22) which become

$$
\left|w-\kappa_{1}\right|_{p} \ll 2^{-t(1+\varepsilon /(2 n))} .
$$

To obtain inequality (23) use the facts that, for $j=1, \ldots, n-1$, all the derivatives satisfy $\left|P_{d}^{(j)}\left(\kappa_{1}\right)\right|_{p} \ll N^{-n(n-j)}$ from Lemma 4 and that the last term (for the $n$th derivative) can be estimated using (10) to obtain $\left|P_{d}(w)\right|_{p} \ll 2^{-t(n+1+\varepsilon /(2 n))}$. Using these new estimates Cases 1, 2 and 3 follow in exactly the same way as before, proving the theorem.

Acknowledgements. We are grateful to the EPSRC that supports the second author and granted the first author a research grant to visit the University of York. The Royal Society provided Jin Yuan with a three month Ex-agreement Study Visit to come to the University of York and H. Dickinson a travel grant to visit Northwest University in China. Finally we thank Maurice Dodson for his advice and support and the referee for some helpful comments.

\section{References}

[1] A. Baker, On a theorem of Sprindžuk, Proc. Roy. Soc. London Ser. A 292 (1966), 92-104.

[2] V. Bernik, Application of Hausdorff dimension in the theory of Diophantine approximation, Acta Arith. 42 (1983), 219-253 (in Russian); English transl.: Amer. Math. Soc. Transl. 140 (1988), 15-44.

[3] V. Bernik and Yu. Melnichuk, Properties of integral polynomials of p-adic variables with a small norm in the disc, Vestnik L'vov. Politekhn. Inst. 182 (1985), 63-64 (in Russian).

[4] A. O. Gel'fond, Transcendental and Algebraic Numbers, GITTL, Moscow, 1952 (in Russian); English transl.: Dover, New York, 1960.

[5] E. Lutz, Sur les approximations diophantiennes linéaires et p-adiques, Actualités Sci. Indust. 1224, Hermann, 1955.

[6] K. Mahler, Über das Mass der Menge aller s-Zahlen, Math. Ann. 106 (1932), 131139.

[7] A. Sprindžuk, A proof of Mahler's conjecture on the measure of the set of $s$ numbers, Izv. Akad. Nauk SSSR Ser. Mat. 29 (1965), 379-436 (in Russian); English transl.: Amer. Math. Soc. Transl. (2) 51 (1966), 215-272. 
[8] A. Sprindžuk, Mahler's Problem in Metric Number Theory, Transl. Math. Monographs 25, Amer. Math. Soc., Providence, 1969.

Institute of Mathematics

Academy of Sciences

Surganova 11

220072 Minsk, Belarus

E-mail: bernik@im.bas-net.by

Department of Mathematics

University of York

Heslington

York, YO1 5DD, England

E-mail: hd3@york.ac.uk
Department of Mathematics Northwest University Xi'an, 710069, Shaanxi P.R. China E-mail: ec@nwu.edu.cn 